\title{
PAPERS
}

\section{Cytokeratin immunoreactivity of intestinal metaplasia at normal oesophagogastric junction indicates its aetiology}

\author{
A Couvelard, J-M Cauvin, D Goldfain, A Rotenberg, M Robaszkiewicz, J-F Fléjou, \\ members of the Groupe d'Etude de l'Oesophage de Barrett
}

Service d'Anatomie Pathologique, Hôpital Beaujon, Clichy, France

A Couvelard

Département d'Information Médicale, CHU Brest, France

J-M Cauvin

Service de

Gastroentérologie, CHG, Dreux, France

D Goldfain

A Rotenberg

Service de

Gastroentérologie, CHU Brest, France M Robaszkiewicz

Service d'Anatomie Pathologique, Hôpital Saint-Antoine, Paris, France

J-F Fléjou

${ }^{\star}$ Members of the GEOB are listed in the appendix.

Correspondence to: Dr A Couvelard, Service d'Anatomie Pathologique, Hôpital Beaujon, F-92118, Clichy cedex, France. anne.couvelard@

bjn.ap-hop-paris.fr

Accepted for publication 1 May 2001

\begin{abstract}
Background and aims-Cytokeratin (CK) 7 and 20 patterns are specific for long and short segments of Barrett's oesophagus but their use has not been assessed in intestinal metaplasia arising in macroscopically normal gastro-oesophageal junction (GOJ).

Patients and methods-This study was carried out in a large prospective series of 254 patients who underwent upper endoscopy, had normal gastro-oesophageal anatomy, and who had biopsies of the antrum, fundus, cardia, GOJ, and lower oesophagus. Intestinal metaplasia of the GOJ was typed by histochemistry with high iron diamine-alcian blue staining and by immunohistochemistry using CK7 and CK20 antibodies. Results were correlated with clinical, endoscopic, and pathological data. Results-Sixty (23.6\%) of our patients presenting with a normal GOJ had intestinal metaplasia. The CK7/CK20 pattern identified two groups of patients: one highly correlated with Barrett's and the other with characteristics of Helicobacter pylori gastritis. The Barrett's type CK7/CK20 pattern was related to a high frequency of gastrooesophageal reflux symptoms $(p<0.02)$ and normal endoscopic appearance of the stomach $(p<0.03)$. In contrast, the gastric type CK7/CK20 pattern was linked to atrophic $\quad(p<0.02)$ or erythematous $(p<0.05)$ appearance of the stomach $(\mathrm{p}<0.03)$, high frequency of $\boldsymbol{H}$ pylori infection $(p<0.04)$, antral inflammation $(p<0.006)$ with atrophy $(p<0.02)$, and intestinal metaplasia $(p<0.02)$.

Conclusion-In patients presenting with intestinal metaplasia in normal appearing GOJ, the cytokeratin pattern identifies two groups of patients, one with features identical to those of long segment Barrett's oesophagus and one with features seen in $H$ pylori gastritis. These data may be used by clinicians and should result in improved endoscopic surveillance strategies targeted specifically at patients at increased risk of Barrett's oesophagus and thus cancer. (Gut 2001;49:761-766)
\end{abstract}

Keywords: Barrett's oesophagus; cardia; intestinal metaplasia; cytokeratins
The incidence of oesophageal adenocarcinomas originating from neoplastic transformation of intestinalised metaplastic mucosa, the so-called Barrett's mucosa commonly thought to be a consequence of gastro-oesophageal reflux disease (GORD), ${ }^{12}$ is increasing. ${ }^{34}$ Identification of Barrett's oesophagus is readily achieved in patients with long segments of intestinal metaplasia by correlating endoscopic and biopsy findings. ${ }^{5}$ Difficulties arise when intestinal metaplasia is present in biopsy specimen taken from the gastro-oesophageal junction (GOJ) with ultrashort segments of columnar mucosa or normal appearing squamocolumnar junction (or $\mathrm{Z}$ line) at endoscopy. It may be impossible in such cases to distinguish intestinal metaplasia of the distal oesophagus, related to GORD, from intestinal metaplasia of the proximal stomach (cardia) related to multifocal Helicobacter pylori gastritis. As no absolute histological criteria for diagnosing Barrett's mucosa have yet been established, the diagnostic implication of intestinal metaplasia at the normal GOJ with regard to diagnosis of ultrashort segment Barrett's oesophagus remains to be defined. Recently, it was shown by Ormsby and colleagues ${ }^{6}$ that the differential reactivity patterns of expression of cytokeratin (CK) subsets 7 and 20 were useful for distinguishing between long or short segment Barrett's mucosa and gastric intestinal metaplasia. ${ }^{67}$ The pattern of immunoreactivity of CK7 and CK20 has not yet been defined at the normal GOJ. We therefore studied 254 patients who underwent upper endoscopy, had normal gastro-oesophageal anatomy, and had biopsies of the antrum, fundus, cardia, GOJ, and oesophagus, to (1) determine the CK7 and CK20 pattern in GOJ biopsy specimens with intestinal metaplasia, and (2) evaluate the relationships between this pattern and demographic, clinical, endoscopic, and histological data.

\section{Methods}

PATIENTS

Patients undergoing gastroscopy from 1998 to 1999 for upper gastrointestinal symptoms, and

Abbreviations used in this paper: $\mathrm{CK}$, cytokeratin; GOJ, gastro-oesophageal junction; GORD, gastro-oesophageal reflux disease; AB-HID, Alcian blue-high iron diamine. 
presenting with an endoscopically normal GOJ were prospectively identified. These patients came from the practice of 11 experienced gastroenterologists working in an academic setting. In each case, the following patient data were obtained: age, sex, weight, and past medical history of ulcer, gastro-oesophageal reflux symptoms (either heartburn or acid regurgitation), and epigastralgia. Drug therapy, smoking history, and alcohol consumption were also recorded.

Exclusion criteria were: past history of oesophagitis or Barrett's oesophagus, prior antibiotic therapy or proton pump inhibitor treatment in the month before endoscopy, prior $H$ pylori eradication, history of gastrooesophageal surgery, and digestive pathology related to portal hypertension.

The study was approved by the ethics committee of the Centre Hospitalier Universitaire de Brest and all patients gave written informed consent prior to inclusion.

ENDOSCOPY AND BIOPSY PROTOCOL

Endoscopy was performed in a standard fashion, with visualisation of the oesophagus, stomach, and duodenum. The appearance of the GOJ was carefully studied in the prograde view and after retroversion in the stomach. The distance from the $\mathrm{Z}$ line to the proximal margin of the gastric mucosal folds was recorded. The $\mathrm{Z}$ line was considered as normal appearing when located no more than $1 \mathrm{~cm}$ above the proximal end of the gastric folds. Patients with columnar lined oesophagus, defined as a columnar epithelium extending $>1 \mathrm{~cm}$ proximal to the gastric folds, were excluded from the evaluation. Patients presenting with grade I or more oesophagitis according to the SavaryMiller classification were excluded. ${ }^{8}$ Hiatal hernia, oesophagitis, gastritis, duodenitis, and gastric or duodenal ulcer were reported as present or absent.

Eleven mucosal biopsy specimen were taken for histological analysis from each patient: one from the oesophagus $1 \mathrm{~cm}$ above the $\mathrm{Z}$ line; four from the squamocolumnar junction (with routine biopsy forceps, paying particular attention so as to obtain a mucosal biopsy directly across the squamocolumnar junction ( $\mathrm{Z}$ line) with one cup of the biopsy forceps positioned on squamous epithelium and the other cup positioned on columnar epithelium); two from the cardia $1 \mathrm{~cm}$ below the $\mathrm{Z}$ line; two from the gastric corpus $8-10 \mathrm{~cm}$ under the cardia; and two from the prepyloric antrum $2-5 \mathrm{~cm}$ above the pylorus.

Biopsy specimens were fixed in $4 \%$ formalin, embedded in paraffin wax, sectioned $(4 \mu \mathrm{m})$ on three levels, stained with haematoxylin-eosin, and mounted on glass slides. In addition, we used Cresyl violet staining to reveal $H$ pylori infection by light microscopy. ${ }^{9}$ Sections were reviewed by a single experienced gastrointestinal pathologist. For each section, the mucosal type was recorded (oesophageal squamous, cardiac, fundic, antral). The type of gastritis and degree of neutrophil infiltration, lamina propria mononuclear cell infiltration, and glandular atrophy were graded according to the Sydney system. ${ }^{10}$
Intestinal metaplasia was recorded as present or absent, confirmed in all cases by evaluation of alcian blue ( $\mathrm{pH}$ 2.5)-high iron diamine (ABHID) stained sections, which showed acid mucin within goblet cells. We verified that both superficial and deep glands were present on immunostained slides for adequate evaluation of immunostaining. The presence of dysplasia was determined with the use of a standardised classification, as defined by Riddell and colleagues. ${ }^{11}$

HISTOCHEMISTRY AND IMMUNOHISTOCHEMISTRY Histological slides from the GOJ biopsy specimens with histological evidence of intestinal metaplasia, defined by the presence of goblet cells in glandular mucosa $(n=60)$, were selected in each case. Only 34 cases with sufficient areas of intestinal metaplasia could be studied for both histochemistry with ABHID staining and immunohistochemistry.

AB-HID staining distinguished three types of intestinal metaplasia: type I, or complete intestinal metaplasia, consisting of absorptive enterocytes alternating with goblet cells; types II and III, or incomplete intestinal metaplasia, consisting of columnar cells alternating with goblet cells. The latter cells contained sialomucins, coloured in blue (type II), or sulphomucins, coloured in black (type III).

Immunostaining was performed on formalin fixed, paraffin wax embedded sections using a three step indirect method for CK7 (clone OV-TL 12/30; Dako, Glostrup, Denmark; dilution 1/40) and CK20 (clone Ks 28.8; Dako; dilution 1/40) monoclonal antibodies. Sections were deparaffinised, heated in a microwave oven, and treated with $0.1 \%$ hydrogen peroxide in methanol before staining. All slides were counterstained with haematoxylin. Positive and negative (obtained by omitting the primary antibody, replaced by Tris buffer saline) controls slides were produced using colonic epithelium.

EVALUATION OF CYTOKERATIN STAINING

Assessment of CK7 and CK20 was undertaken by noting the distribution and intensity of cytoplasmic CK7 and CK20 staining in intestinal metaplasia in all GOJ biopsy specimens. Slides were coded such that the observer was blind to the clinical and endoscopic data and to the histological appearance of the biopsy specimens from other sites. Evaluation of CK staining was determined in a semiquantitative fashion, as described by Ormsby and colleagues ${ }^{6}$ : intestinal metaplasia with a Barrett type CK7/CK20 pattern (Barrett CK7/CK20 pattern) was characterised by strong diffuse CK7 (fig 1A) and superficial CK20 (fig 1B) immunostaining. Intestinal metaplasia with a gastric type CK7/ CK20 pattern (gastric CK7/CK20 pattern) depended on the histochemical type of intestinal metaplasia: complete metaplasia was characterised by absent CK7 (fig 2A) immunostaining and strong diffuse CK20 (fig 2B) immunostaining whereas incomplete metaplasia was characterised by weak patchy CK7 staining and moderate patchy CK20 immunostaining. 

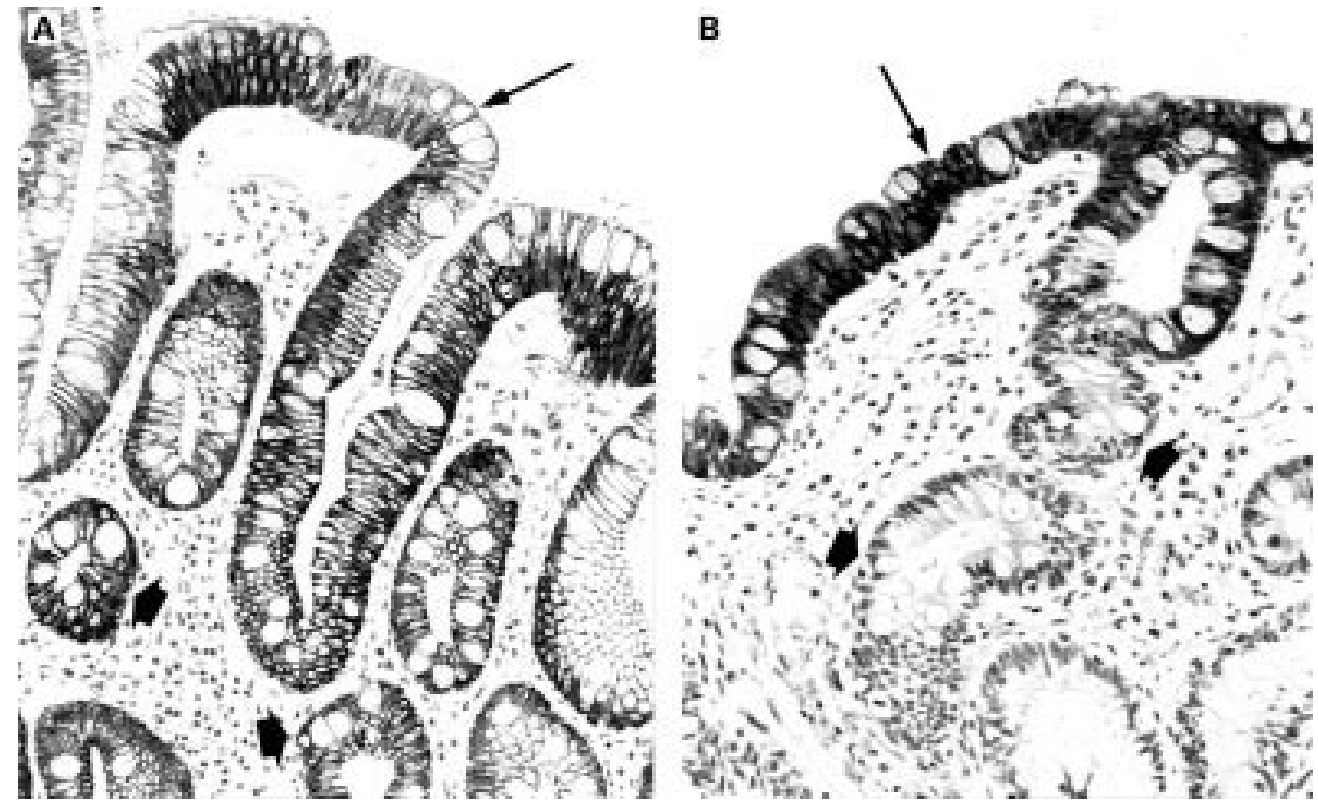

Figure 1 Barrett's cytokeratin 7 and 20 (CK7/CK20) pattern in biopsy specimens from the $Z$ line with incomplete (A) and complete (B) intestinal metaplasia. (A) Diffuse strong CK7 staining of surface epithelium (arrow) and deep glands (arrowheads). Immunoperoxidase with nuclear counterstain by Mayer's haematoxylin (original magnification $\times 200)$. (B) Band-like CK20 staining of superficial epithelium (arrow). Absent immunoreactivity of deep glands (arrowheads).

Immunoperoxidase with nuclear counterstain by Mayer's haematoxylin (original magnification $\times 200$ ).

STATISTICAL ANALYSIS

Statistical analysis was performed using the $\chi^{2}$ or Fisher's test when appropriate, and the Kruskal-Wallis test for quantitative data.

\section{Results}

The study population comprised 254 patients with an endoscopically normal GOJ for final evaluation (121 men and 133 women; mean age 49 (15) years (range 18-93)). In 60 patients (24\%; 95\% confidence interval $18-29 \%$ ), the biopsy specimen from the GOJ showed intestinal metaplasia with goblet cells. Among the 60

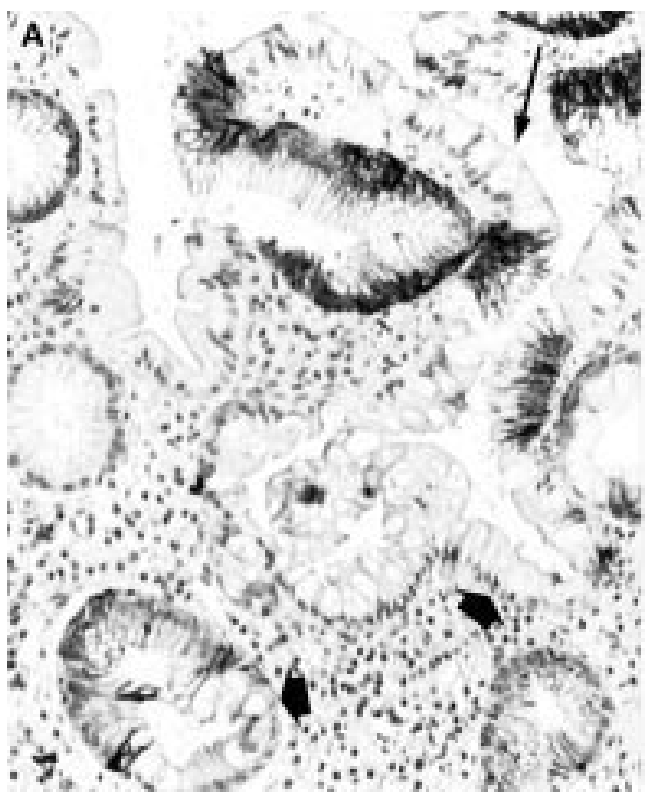

patients, biopsy specimens from the $\mathrm{Z}$ line contained both squamous and columnar epithelium in 48 cases $(80 \%)$ and columnar epithelium in only 12 cases $(20 \%)$. No cases presented with low or high grade dysplasia. Thirty four of these cases with sufficient area of intestinal metaplasia were further evaluated for histochemistry and immunohistochemistry. The 26 other patients were excluded from the evaluation.

HISTOCHEMICAL RESULTS

Twenty seven patients presented with type I complete intestinal metaplasia; 13 patients

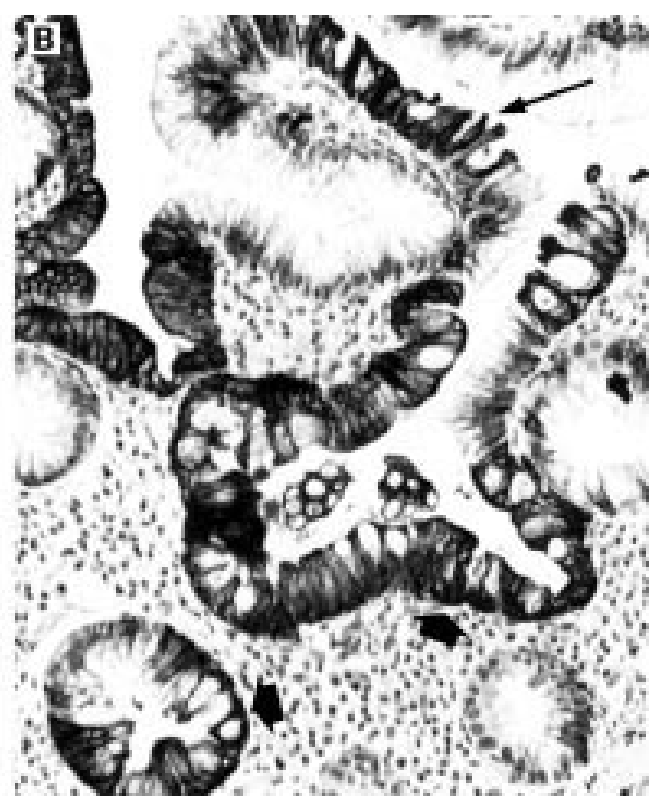

Figure 2 Gastric cytokeratin 7 and 20 (CK7/CK20) pattern in a biopsy specimen from the $Z$ line with complete intestinal metaplasia. (A) Absent CK7 staining of superficial epithelium (arrow) and deep glands (arrowheads). Immunoperoxidase with nuclear counterstain by Mayer's haematoxylin (original magnification $\times 200$ ). (B) Strong diffuse CK20 immunostaining of both superficial epithelium (arrow) and deep glands (arrowheads). Immunoperoxidase with nuclear counterstain by Mayer's haematoxylin (original magnification $\times 200$ ). 
presented with incomplete intestinal metaplasia. Complete intestinal metaplasia was associated with incomplete intestinal metaplasia in six cases.

Two groups of patients were compared: (a) patients presenting with complete intestinal metaplasia only $(n=21)$ and (b) patients presenting with incomplete intestinal metaplasia, associated or not with complete intestinal metaplasia $(n=13)$. These two groups were compared regarding patient age, sex, symptoms, endoscopic findings, histological evaluation of $H$ pylori infection, inflammation, intestinal metaplasia, and atrophy of the GOJ, cardia, antrum, and fundus. The only statistically significant difference between the two groups of patients was the frequency of gastrooesophageal reflux: $2 / 21$ (9\%) patients with complete intestinal metaplasia presented with symptoms of reflux compared with $6 / 13(46 \%)$ with incomplete intestinal metaplasia $(\mathrm{p}<0.03)$.

\section{IMMUNOHISTOCHEMICAL RESULTS}

Among the 27 cases of complete intestinal metaplasia, eight were of Barrett's pattern and 19 were of the gastric pattern. Among the 13 cases of incomplete intestinal metaplasia, 11 were of Barrett's pattern and two were of the gastric pattern. Complete and incomplete intestinal metaplasia were associated in two cases with the gastric pattern and in four cases with Barrett's pattern.

Two groups of patients were compared: (a) patients presenting with the gastric CK $7 / \mathrm{CK} 20$ pattern $(\mathrm{n}=18)$ and (b) patients presenting with Barrett's CK7/CK20 pattern $(n=16)$. These two groups were compared regarding patient age, sex, symptoms, endoscopic findings, histological evaluation of $H$ pylori infection, inflammation, intestinal metaplasia, and atrophy of the GOJ, cardia, antrum, and fundus. Results are summarised in table 1 .

Comparing the demographic and clinical findings, the only statistically significant difference between the two groups of patients was a higher frequency of gastro-oesophageal reflux symptoms in patients presenting with Barrett's CK7/CK20 pattern $(p<0.02)$. No significant differences were found for other findings such as age, sex $(p=0.07)$, weight, smoking history, or alcohol consumption.

Table 1 Comparison of clinical, endoscopic, and histological features in intestinal metaplasia of the gastro-oesophageal junction (GOF) with Barrett's CK7/CK20 pattern or the gastric CK7/CK20 pattern

\begin{tabular}{lcll}
\hline & $\begin{array}{l}\text { Barrett's CK7/CK20 } \\
(n=16)(n(\%))\end{array}$ & $\begin{array}{l}\text { Gastric CK7/CK20 } \\
(n=18)(n(\%))\end{array}$ & p Value \\
\hline Clinical findings & & & \\
$\quad \begin{array}{l}\text { Reflux symptoms } \\
\text { Male gender }\end{array}$ & $7(43)$ & $1(5.5)$ & $<0.02$ \\
$\begin{array}{l}\text { Gastric endoscopic aspect } \\
\text { Normal }\end{array}$ & $12(75)$ & $8(44)$ & $\mathrm{NS}$ \\
$\quad$ Erythematous & $10(62.5)$ & $8(44)$ & $<0.03$ \\
Atrophic & $1(6.25)$ & $7(38.8)$ & $<0.05$ \\
Histological feature & $2(12.5)$ & $9(50)$ & $<0.02$ \\
$\quad \begin{array}{l}\text { GOJ H pylori } \\
\text { Cardia H pylori }\end{array}$ & $5(31.2)$ & $12(66)$ & $<0.04$ \\
$\begin{array}{l}\text { Antral chronic gastritis } \\
\text { Antral atrophy }\end{array}$ & $5(31.2)$ & $12(66)$ & $<0.006$ \\
Antral intestinal metaplasia & $8(50)$ & $17(94.4)$ & $<0.02$ \\
\hline
\end{tabular}

NS, not significant.
Comparing endoscopic findings, patients with Barrett's CK7/CK20 pattern were more likely to have a normal appearance of the stomach $(p<0.03)$ whereas those with the gastric $\mathrm{CK} 7 / \mathrm{CK} 20$ pattern were more likely to have a erythematous or atrophic appearance $(p<0.05$ and $p<0.02$, respectively). No significant differences were found regarding hiatal hernia.

Comparing histological data, significant differences were found between patients presenting with Barrett's CK7/CK20 pattern relative to those presenting with the gastric CK7/CK20 pattern. $H$ pylori infection of the GOJ and cardia was found with a higher frequency in patients presenting with intestinal metaplasia of the gastric CK7/CK20 pattern $(\mathrm{p}<0.04)$. Antral gastritis, with glandular atrophy and intestinal metaplasia, were more frequent in the group of patients presenting with the gastric CK7/CK20 pattern $(\mathrm{p}<0.006, \mathrm{p}<0.02$, and $\mathrm{p}<0.02$, respectively).

\section{Discussion}

Our results show that (1) $24 \%$ of patients presenting with a macroscopically normal GOJ have intestinal metaplasia, (2) the CK7/CK20 pattern can differentiate these patients into two groups: those highly correlated with Barrett's oesophagus and those with characteristics of $H$ pylori gastritis.

Our observation that $24 \%$ of patients with an endoscopically normal GOJ have intestinal metaplasia is in accordance with most previously reports. ${ }^{12-19}$ However, there are differences in the prevalence of intestinal metaplasia in these studies, ranging from $5 \%{ }^{12}$ to $27 \% .{ }^{13}$ In the absence of a clearly recognisable segment of Barrett's mucosa, and considering the difficulties in defining the normal anatomy of the GOJ, it may be impossible to precisely determine whether or not intestinal metaplasia develops from the distal oesophagus, and thus is related to GORD, or from the proximal stomach secondary to $H$ pylori infection. The potential contributions of both causes are unclear. Indeed, recent studies focusing on the pathogenesis and epidemiology of cardiac inflammation and intestinal metaplasia have yielded contradictory results. Investigators have found a strong association between carditis and GORD but no association between carditis and $H$ pylori infection. ${ }^{18-21}$ These studies argue for short segment Barrett's being the result of upward extension of intestinal metaplasia at the GOJ mucosa. In contrast, other investigators concluded that inflammation and intestinal metaplasia at the normal GOJ are related to $H$ pylori infection. ${ }^{1622}$ Differences in patient population, number of biopsy specimens, and criteria to identify and sample the cardiac region may contribute to these disparate results. Goldstein and Karim, in a recent study of 150 patients presenting with a normal GOJ anatomy, showed that cardiac inflammation and intestinal metaplasia were related to oesophageal squamous inflammation in non- $H$ pylori infected patients, and to antral inflammation and cardia $H$ pylori infection in $H$ pylori 
infected patients. ${ }^{13}$ Their results clearly demonstrate that inflammation and intestinal metaplasia at the squamocolumnar junction may be observed in Barrett's oesophagus and in $H$ pylori multifocal gastritis. Moreover, they show on biopsy follow up that inflammation is linked to intestinal metaplasia and that a reduction in inflammation may allow the metaplastic mucosa to revert to normal. ${ }^{23}$ Finally, since absolute histological criteria for diagnosing Barrett's mucosa have not yet been established, none of the studies discussed above provided histological data that might have been useful in suggesting a columnar or squamous origin for the metaplastic tissue.

Some authors have discussed histochemical typing of intestinal metaplasia for the diagnosis of Barrett's oesophagus. Patients with long segment Barrett's oesophagus have been noted to have a high prevalence of incomplete intestinal metaplasia in the oesophagus. ${ }^{124-28}$ However, incomplete intestinal metaplasia is not a sensitive or specific marker of Barrett's oesophagus as up to $45 \%$ of intestinal metaplasias in the stomach show areas of incomplete type..$^{29}$ The frequency of the different histochemical types of intestinal metaplasia from normal GOJ and their significance are still the subject of debate. Peuchmaur et al pointed out that incomplete intestinal metaplasia was present in biopsy specimens taken from the normal GOJ but their patients all presented with symptoms of reflux oesophagitis. ${ }^{25}$ Günther et al have characterised histochemically intestinal metaplasia from biopsy specimens taken from the GOJ in a large series of 443 patients presenting as endoscopically normal $(n=385)$ or with a suspicion of Barrett's mucosa $(2 \mathrm{~cm}$ above the gastric mucosal folds or short mucosal tongues, $\mathrm{n}=58) .{ }^{17}$ They found that complete intestinal metaplasia was associated with an endoscopically unremarkable $\mathrm{Z}$ line and with multifocal $H$ pylori gastritis whereas incomplete intestinal metaplasia was associated with a $\mathrm{Z}$ line suspected of Barrett's oesophagus. Voutilainen et al showed in a large series of 1058 patients that complete intestinal metaplasia at the oesophagogastric junction is associated with multifocal atrophic gastritis whereas incomplete intestinal metaplasia is associated with carditis and gastro-oesophageal reflux. ${ }^{31}$ In the present study, we found that type I complete intestinal metaplasia was the most frequent type in normal appearing GOJ, representing $62 \%$ of our cases ( 21 of 34 patients). The only significant difference between patients presenting with complete or incomplete type intestinal metaplasia was the higher frequency of gastrooesophageal reflux symptoms in the latter group. We failed to find any association between complete and incomplete intestinal metaplasia regarding any other clinical symptom, or endoscopic or histological finding. Our results suggest that, as reported in long segment Barrett's oesophagus, the histochemical status of intestinal metaplasia is insufficiently sensitive to be useful in identifying the oesophageal or gastric origin of intestinal metaplasia arising in ultrashort tongues of columnar mucosa or normal appearing $\mathrm{Z}$ line.
In contrast, using CK7/CK20 immunoreactivity, we identify two populations that may have a distinct origin of intestinal metaplasia, one with hallmark features of GORD and the other correlated with $H$ pylori induced gastritis. Ormsby et al have defined two different CK7/CK20 immunoreactivity patterns that were strongly associated with long segments of Barrett's oesophagus (sensitivity $97 \%$, specificity $100 \%$ ) and gastric intestinal metaplasiathat is, Barrett's CK7/CK20 pattern and the gastric CK7/CK20 pattern, respectively. ${ }^{6}$ In a recent study they demonstrated that Barrett's CK7/CK20 pattern identified a subset of patients with an endoscopically suspected short segment Barrett's oesophagus who had a patient profile similar to that seen in long segment Barrett's oesophagus. ${ }^{7}$ In this study, short segments were defined as tongues and/or circumferential columnar appearing mucosa $<3 \mathrm{~cm}$ above the gastric folds, with exclusion of normal or serrated $\mathrm{Z}$ line $<0.5 \mathrm{~cm}$ proximally. We have confirmed in our study that the two patterns of expression were readily identified using endoscopic biopsy specimens taken from normal appearing GOJ, with exclusion of serrated $Z$ line $>1 \mathrm{~cm}$ proximally. We identified two groups of patients: the first group (16 of 34) had a Barrett's CK7/CK20 pattern and features of Barrett's oesophagus, including gastro-oesophageal reflux symptoms, normal gastric appearance and a low frequency of $H$ pylori infection. Despite a preponderance for male patients in this subgroup, the sex difference was not significant. In contrast, the second group (18 of 34) had a gastric CK7/CK20 pattern and features similar to those seen in $H$ pylori gastritis, including antral chronic gastritis with atrophy and intestinal metaplasia, and high frequency of $H$ pylori infection. Our results suggest that the main causes of intestinal metaplasia in biopsy specimens taken from normal GOJ (that is, $H$ pylori or GORD) determine the cytokeratin pattern of expression.

Early recognition of intestinal metaplasia with Barrett's CK7/CK20 pattern could be important, together with clinical and endoscopic data, in identifying patients that may have an increased risk of developing Barrett's oesophagus. This may have considerable clinical significance as Barrett's oesophagus is an important risk factor for the development of dysplasia and adenocarcinoma. ${ }^{12} 32$ Moreover, some studies have shown a relatively high frequency of dysplasia in intestinal metaplasia of normal appearing $\mathrm{GOJ},{ }^{33}$ a result that is still debated. ${ }^{34}$ Our data may result in improved endoscopic surveillance strategies targeted specifically to these patients at increased risk of Barrett's oesophagus, dysplasia, and thus cancer. However, long term follow up of our patients is needed to confirm and quantify this risk.

We thank the "Société Nationale Française de GastroEntérologie", SNFGE, and the "Association Charles Debray" for their financial support. 


\section{Appendix}

MEMBERS OF THE “GROUPE D'ETUDE DE L'OESOPHAGE DE BARRETT" (GEOB)

Pathologists: Anne Croué (Angers), Alain Volant (Brest), Marie-Danielle Diebold (Reims), Christiane Vissuzaine (Paris), Christine Sagan (Nantes). Gastroenterologists: Jean Boyer (Angers), Guillaume Cadiot (Reims), Michel Mignon (Paris), Thomas Aparicio (Paris), Jean-Paul Galmiche (Nantes), Marc Le Rhun (Nantes).

1 Haggitt RC. Barrett's esophagus, dysplasia and adenocarcinoma. Hum Pathol 1994;25:982-93.

2 Spechler SJ, Robbins AA, Rubin HB. Adenocarcinoma and Barrett's esophagus: an overrated risk? Gastroenterology 1984;87:927-33.

3 Blot WJ, Devesa SS, Kneller RW, et al. Rising incidence of adenocarcinoma of the esophagus and gastric cardia. aAMA 1991;265:1287-9.

4 Pera M, Cameron AJ, Trastek VF, et al. Increasing incidence of adenocarcinoma of the esophagus and esophagogastric junction. Gastroenterology 1993;104:510-13.

5 Cameron AJ, Lomboy CT. Barrett's esophagus: age, prevalence and extent of columnar epithelium. Gastroenter ology 1992;103:1241-5

6 Ormsby AH, Goldblum JR, Rice TW, et al. Cytokeratin subsets can reliably distinguish Barrett's esophagus from intestinal metaplasia of the stomach. Hum Pathol 1999;30:288 94.

7 Ormsby AH, Vaezi MF, Richter JE, et al. Cytokeratin immunoreactivity patterns in the diagnosis of shortsegment Barrett's esophagus. Gastroenterology 2000;119: 683-90.

8 Ollyo JB, Lang F, Fontolliet C, et al. Savary-Miller's new endoscopic grading of reflux oesophagitis: a simple reproductible logical and useful classification. Gastroenterol ogy 1990;98:A100.

9 Burnett RA, Brown IL, Findlay J. Cresyl fast violet staining method for campylobacter like organisms. f Clin Pathol method for campyl

10 Dixon MF, Genta RM, Yardley JH, et al. Classification and grading of gastritis. The updated Sydney system. Am $\mathcal{F}$ Surg 6;20:1161-81.

11 Riddell RH, Goldman H, Ransohoff DF, et al. Dysplasia in inflammatory bowel disease: standardized classification with provisional clinical applications. Hum Pathol 1983;14: 931-68.

12 Hirota WK, Loughney TM, Lazas DJ, et al. Is Helicobacter pylori associated with specialized intestinal metaplasia of the esophagus or stomach? A prospective study of 889 patients. Gastroenterology 1997;112:A149.

13 Goldstein NS, Karim R. Gastric cardia inflammation and intestinal metaplasia: associations with reflux esphagitis and Helicobacter pylori. Mod Pathol 1999;12:1017-24

14 Spechler SJ, Zeroogian JM, Antonioli DA, et al. Prevalence of metaplasia at the gastro-esophageal junction. Lance of metaplasia at

15 Dias Pereira A, Suspiro A, Chaves P, et al. Short segments of Barrett's epithelium and intestinal metaplasia in normal Barrett's epithelium and intestinal metaplasia in normal appearing oesophagogastric junctions:

16 Goldblum JR, Vicari JJ, Falk GW, et al. Inflammation and intestinal metaplasia of the gastric cardia: the role of the gastrooesophageal reflux and H. pylori infection. Gastroenterology 1998;114:663-9.
17 Günther T, Hackelsberger A, Malfertheiner P, et al. Is typing of metaplasia at the squamocolumnar junction revealing its aetiology? Virchows Arch 2000;436:6-11.

18 Hackelsberger A, Günther T, Schultze V, et al. Intestinal metaplasia at the gastrooesophageal junction: Helicobacter pylori gastritis or gastrooesophageal reflux disease? Gut 1998;43:17-21.

19 Trudgill NJ, Suvarna SK, Kapur KC, et al. Intestinal metaplasia at the squamocolumnar junction in patients attending for diagnostic gastroscopy. Gut 1997;41:585-9.

20 Csendes A, Smok G, Burdiles P, et al. "Carditis": an objective histological marker for pathologic gastroesophageal reflux disease. Dis Esophagus 1998;11:101-5.

21 Öberg S, Peters JH, DeMeester TR, et al. Inflammation and specialized intestinal metaplasia of cardia mucosa is a manifestation of gastroesophageal reflux disease. Ann Surg 1997;226:552-32.

22 Chen YY, Antonioli DA, Spechler SJ, et al. Gastroesophageal reflux disease versus Helicobacter pylori infection as the cause of gastric carditis. Mod Pathol 1998;11:950-6.

23 Goldstein NS. Gastric cardia intestinal metaplasia: biopsy follow up of 85 patients. Mod Pathol 2000;13:1072-9.

24 Jass JR. Mucin histochemistry of the columnar epithelium of the oesophagus: a retrospective study. $\mathcal{F}$ Clin Pathol 1981;34:866-70.

25 Peuchmaur M, Potet F, Goldfain D. Mucin histochemistry of the columnar epithelium of the oesophagus (Barrett's oesophagus): a prospective biopsy study. $\mathcal{f}$ Clin Pathol 1984;37:607-10.

26 Haggitt RC, Reid BJ, Rabinovitch PS, et al. Barrett's esophagus: correlation between mucin histochemistry, flow cytometry, and histologic diagnosis for predicting increased cancer risk. Am f Pathol 1988;131:53-61.

27 Lapertosa G, Baracchini P, Fulcheri E. Mucin histochemical analysis in the interpretation of Barrett's esophagus: results of a multicenter study. The operative group for the study of esophageal precancer. Am f Clin Pathol 1992;98: 61-6.

28 Torrado J, Ruiz B, Garay MS, et al. Blood-group phenotypes, sulfomucins, and Helicobacter pylori in Barrett's esophagus. Am $\mathcal{F}$ Surg Pathol 1997;21:1023-9.

29 Ormsby AH, Goldblum JR, Rice TW, et al. The sensitivity and specificity of histologic features in the diagnosis of Barrett's esophagus on routine endoscopic biopsy specimens. Am f Clin Pathol 1999;112:A 533.

30 Sipponen P, Seppala K, Varis K, et al. Intestinal metaplasia with colonic-type sulphomucins in the gastric mucosa; its association with gastric carcinoma. Acta Pathol Microbiol Scand 1980;A88:217-24.

31 Voutilainen M, Färkkilä $M$, Juhola $M$, et al. Complete and incomplete intestinal metaplasia at the eosophagogastric juntion: prevalences and associations with endoscopic erosive oesophagitis and gastritis. Gut 1999;45:644-8.

32 Hamilton SR, Smith RRL. The relationships between columnar epithelial dysplasia and invasive adenocarcinoma arising in Barrett's esophagus. Am f Clin Pathol 1987;87: 301-12.

33 Csendes A, Smok G, Burdiles P, et al. Prevalence of Barrett's esophagus by endoscopy and histologic studies: a prospective evaluation of 306 control subjects and 376 patients with symptoms of gastroesophageal reflux. Dis Esophagus 2000;13:5-11.

34 Voutilainen M, Färkkilä M, Mecklin JP, et al. Classical Barrett esophagus contrasted with Barrett-type epithelium at normal-appearing esophagogastric junction. Scand $\mathcal{f}$ Gastroenterol 2000;35:2-9. 